\title{
Time Synchronization with White Rabbit - Experience from Tunka-HiSCORE
}

\author{
Ralf Wischnewski* \\ DESY, Zeuthen \\ E-mail: ralf.wischnewski@desy.de
}

\section{Martin Brueckner}

PSI, Villigen

E-mail: martin.bruecknerepsi.ch

\section{Andrea Porelli}

\section{DESY, Zeuthen}

E-mail: andrea.porelli@desy.de

Upcoming Gamma-Ray and Cosmic-Ray experiments require relative time calibration of all detector components with (sub-)nanosecond precision. White Rabbit, a new technology for time and frequency transfer, can be applied here.

We describe a White Rabbit (WR) based design for trigger time-stamping, originally developed for Tunka-HiSCORE - a timing array for Gamma-Ray astronomy now under construction. Subnsec synchronization results from cosmic ray shower data, in-situ artifical light source calibrations and laboratory tests taken over several years are presented.

The 34th International Cosmic Ray Conference,

30 July- 6 August, 2015

The Hague, The Netherlands

\footnotetext{
* Speaker.
} 


\section{Introduction}

We describe a modern timing system for astroparticle physics experiments, customized as a prototype for the Tunka-HiSCORE array. Tunka-HiSCORE is a large area wide angle detector for gamma rays from $20 \mathrm{TeV}$ to few $\mathrm{PeV}$, and cosmic rays above few $\mathrm{PeV}$, under construction in the Tunka Valley, Siberia [1]. It is a non-imaging atmospheric Cherenkov light-front sampling array, covering an area of up to $100 \mathrm{~km}^{2}$.

For precision reconstruction of the atmospheric air shower direction, Cherenkov light arrival times at all detector stations have to be measured with (sub-) nsec relative timing precision. To reach this we used - for the first time in a large astroparticle physics experiment - the new White Rabbit technolgy for precision time and frequency transfer [3]; thus avoiding the substantial design and construction effort for a custom-made solution.

This paper summarizes the laboratory test and long-term field experience, gained with the White Rabbit setups for the HiSCORE prototype timing system from 2012-2015. Section 2 briefly introduces the White Rabbit technology; section 3 describes the various setups used at HiSCORE, their methodics and physics results. We conclude, that White Rabbit fulfills all requirements for precision timing in next generation large experiments like CTA [4]. White Rabbit has the potential to become a standard technology in this field ("time-synchronization out of the box").

\section{Clock distribution and time-stamping with White Rabbit}

Figure 1(a) gives a typical White Rabbit (WR) setup [3,5]. The baseline ingredients are (1) WR-Switches (WRS) and (2) WR-Nodes, connected by standard Gigabit Ethernet fibers. The WRS are arranged like in a normal ethernet-network; the central WRS (Grand Master Switch) acts as the time source (e.g. connected to a GPS antenna).

White Rabbit is build on Gigabit Ethernet (1000base-BX10) and takes advantage of the Ethernet standards SyncE and Precision Time Protocol. It offers sub-ns precision, with excellent clock phase stability. It utilizes one fiber for each WR-node for both synchronization and user data, and compensates dynamically for clock drifts due to e.g. environmental influences (temperature).

The WR-node allows to interface the user system (eg. the DAQ of a detector station or a telescope) to the WR-time system: by either time-stamping signals from the detector and/or by supplying clock-information (like PPS or periodic clock signals) to the detector, as shown for the lower WR-node in fig.1 (a) by "trigger" and "clock" signals. As the WR-node device we use for this work the "Simple PCIe FMC carrier" (SPEC), shown in fig.1(b) - a reliable workhorse of the WRcommunity [6]. It has a Spartan-6 FPGA (with the WR PTP Core, optional custom firmware and software) and can accommodate FMC-mezzanine cards. We use the FMC-DIO5Ch, a 5 channel digital I/O card, for analog/digital trigger input, control signals, and PPS/MHz clock output (e.g. for clock performance tests).

We summarize the main arguments to decide for White Rabbit:

- Clock-driven architecture: precision clocks are localized inside the front-end stations (nodes)

- Time stamping at front-end enables complex digital trigger schemes (next neighbor or array)

- Availability and commercial support for all main components

- Open source approach (firmware/software), good documentation and community support 


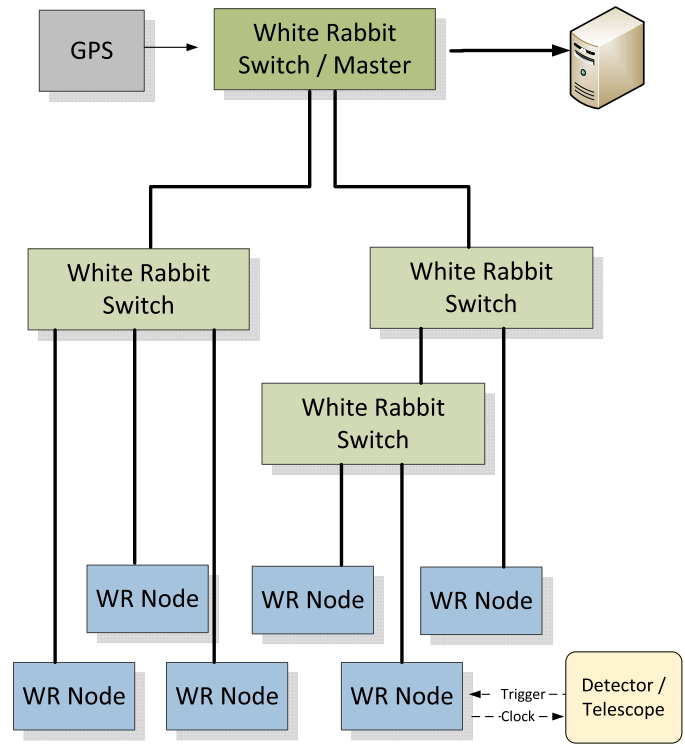

(a)

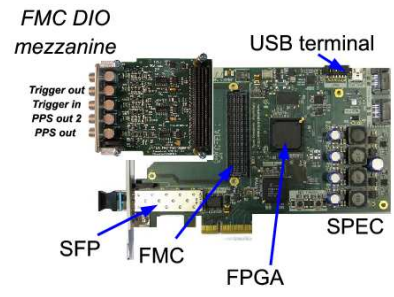

(b)

Figure 1: (a) The White Rabbit network, made up of WR-switches (Grand Master and normal WRS) and of WR-nodes. The WR-nodes can deliver clock-signals to, and/or extract trigger timestamps from the associated detectors, as symbolized for the lower-right WR-node (see text). (b) Example of a WR-node: The SPEC card, the WR-node used for this work. The precision time, kept on the Spartan-6 FPGA is synchronized through the fiber cable (SFP) to the central WR-switch.

- Application interfacing can be reduced to simple, passive FMC mezzanines

- Design simplicity and flexibility for even large scale setups

- Detailed calibration procedures and online performance monitoring supported

- Cost- and time-efficiency compared to custom-made solutions (manpower and investment).

\section{Experimental setups at HiSCORE}

Tunka-HiSCORE [1, 2] is a non-imaging atmospheric Cherenkov light-front sampling array, build of many optical detector stations, located at typical distances of $100-200 \mathrm{~m}$. The detector is under construction, it will cover an area of $1 \mathrm{~km}^{2}$ in the inital, and up to $100 \mathrm{~km}^{2}$ in the final phase.

\subsection{The HiSCORE-SPEC and Laboratory tests}

To apply White Rabbit for trigger time-stamping in HiSCORE, the standard performance of the SPEC-node has been extended. As presented in [5], the SPEC FPGA-design was modified and allows now to

(1) time stamp external digital trigger signals with ns-precision, and transfer the time-stamps and counter information via WR-fiber to the WR-master; or

(2) form the trigger decision on the WR-node by ns-sampling of an analog input signal, dicriminated against a comparator threshold; a trigger being generated after $\geq \mathrm{N}$ consequtive ns-samples 
being high (typically set to $9 \mathrm{~ns}$ ). The trigger signal is time-stamped and transported, like for (1); additional DAQ-I/O signals are generated, see [5, 8].

Laboratory tests of a WR-system, with all components located closeby, offer the possibility to ultimatively check the clock performance (precision, resolution) for an ensemble of WR-nodes by comparing them directly against each other or to precisely defined reference signals (all brought by direct cable connections). With such "table-top" setups, including climate chamber temperature tests (fiber: $-20 \ldots+40^{\circ} \mathrm{C}$; SPEC: $0 \ldots+30^{\circ} \mathrm{C}$ ) the basic timing precisison (clock jitter) was measured to be better than $\sigma_{W R} \sim 0.2 \mathrm{~ns}$ [5]. A recent precision measurement with a HydraHarp-400 setup by PicoQuant [11] with picosecond event timing precision, shown in figure 2(a), gives a time jitter for two SPEC-nodes $\sigma_{W R}<60 \mathrm{ps}$. Also, the stability of the nsec-trigger-stamping is excellent. The result for the "jitter" of the digital time-stamps from two WR-SPECs is shown in fig.2(b) [5]. All measurements are in agreement with a clock precision better than $0.15 \mathrm{~ns}$ per WR-node for the full temperature range investigated.

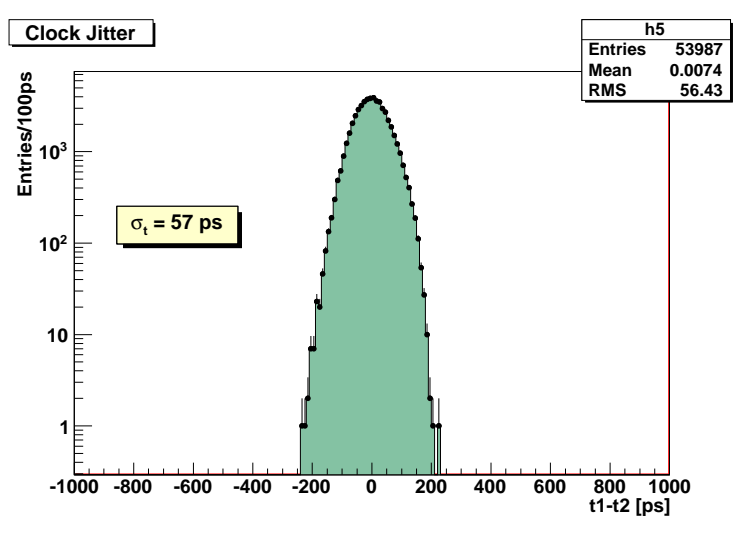

(a)

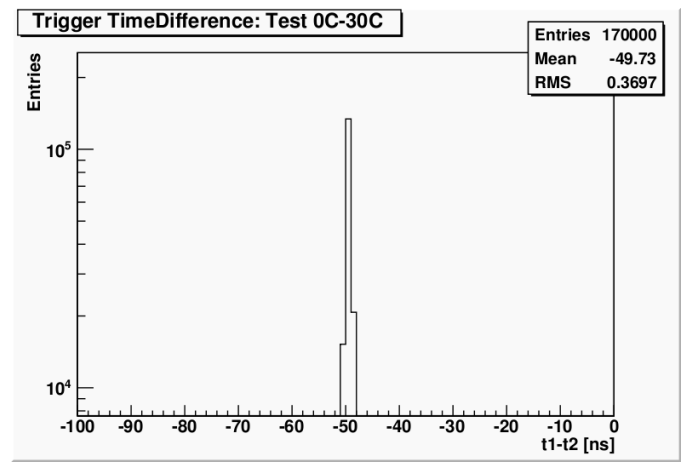

(b)

Figure 2: Sub-nsec precision WR-timing in laboratory tests. (a) Clock phase stability: Distribution of time difference between PPS clock-pulses from two WR-nodes (SPEC1/2), for a 15-hour run with a HydraHarp-400 setup. (b) Trigger stamping: Difference of digital trigger time-stamps (1 ns resolution) of the PPS pulses from SPEC1/2. SPEC2 and the $500 \mathrm{~m}$ fiber-coil were subjected to climate chamber temperature ramps between $0^{\circ}$ and $30^{\circ} \mathrm{C}$. For details see [5] (time-offset $\neq 0$ due to cabeling).

\subsection{Setups at Tunka-HiSCORE}

We report results from various HiSCORE setups, that operated between 2012 and 2015.

\section{- Baseline tests}

With the HiSCORE-3 prototype array (winter season 2012/13) we deployed WR-nodes in each of the three stations - mainly for methodical tests, as shown in figure 3. Clock phase stability and trigger stamping performance between different stations, and for independent (redundant) WR-nodes located inside a single station was obtained. With a "table-top"-like setup in the data-center, the temperature dependent fiber-delay compensation was studied 


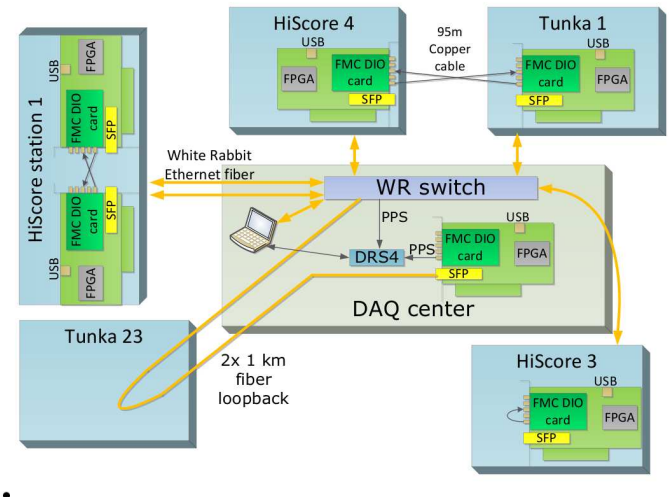

(a)

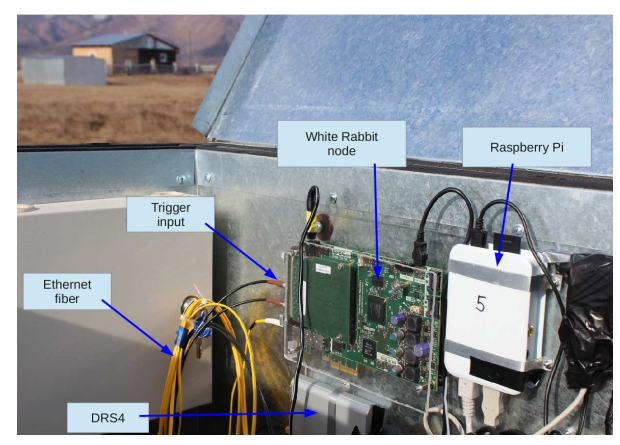

(b)

Figure 3: (a) The complex HiSCORE WR field-setup, as operated in 2012/13 to evaluate timesynchronization and nsec-trigger time-stamping by monitoring WR-nodes [7]. (b) HiSCORE prototype station (2012/13) with DAQ and WR components.

(long fiber to Tunka-23), for details see [7, 10]. Note the "monitoring WR-node" as in Station-1 (fig.3(a)) - an example of a possible verification configuration, when there is no external, independent source of precision time signals to be fed to the stations (WR-nodes).

\section{- Physics Setup: HiSCORE-9}

The 9-station array HiSCORE-9, operating over the winter-season 2013/14, was the first astroparticle physics setup using WR for longterm operation (see sects. 3.3 and 3.4 below). Two independent DAQ-systems were build for HiS-9 [2, 8]. The WR-based DAQ-2 is outlined in fig.4; emphasis was on an end-to-end functional test of the WR time-synchronization functionality with a prototype DAQ.

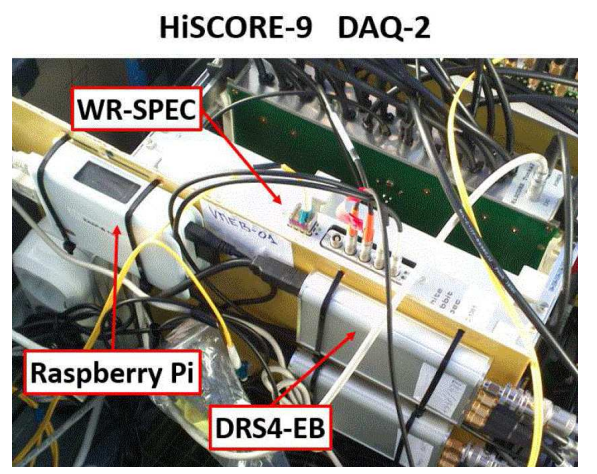

(a)

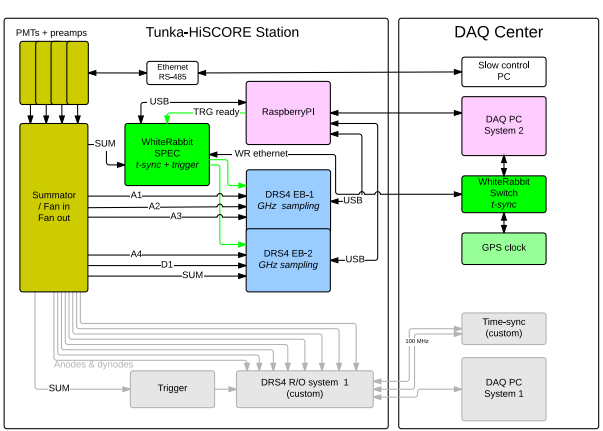

(b)

Figure 4: The HiSCORE-9 DAQ-2 system, with DRS4-Evaluation Board and White-Rabbit timing system. (a) Station setup (with DRS4-EB, WR-SPEC and MiniPC). (b) Schematics of the setup in Station and Center for DAQ-2 (DAQ-1 in grey). See also [8]. 


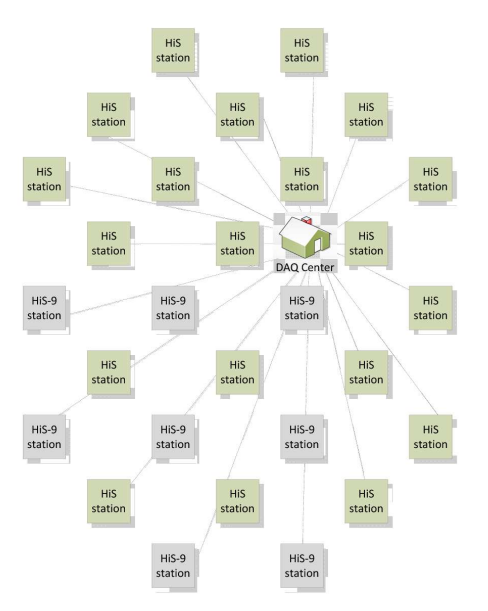

Figure 5: HiSCORE-28 array layout: 28 stations at $100 \mathrm{~m}$ spacing forming a super-cell structure, on a total area of $450 \mathrm{~m} \times 600 \mathrm{~m}$. The HiS-9 stations are indicated, as well as the DAQ-center.

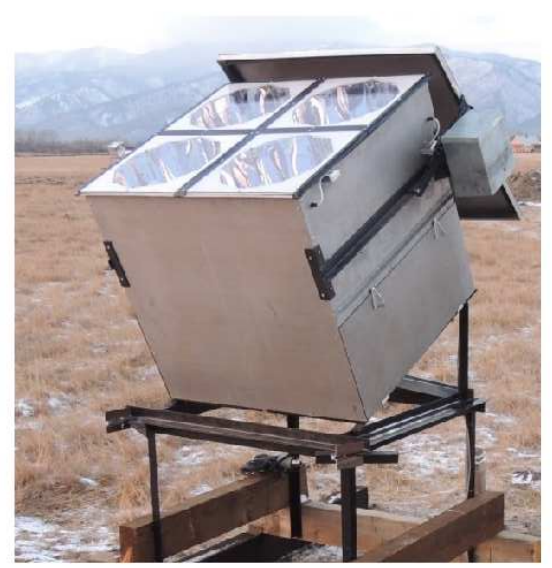

Figure 6: A HiSCORE optical station, with four 8 " PMTs and Winston Cones, inclined by $25^{\circ}$ southwards.

\section{- Physics Setup: HiSCORE-28}

HiSCORE-9 was upgraded in 2014 to the HiSCORE-28 array [2], see figs. 5 and 6. The HiS-28 DAQ combines the DAQ-1 timing system with the WR-SPECs, which time-stamp all DAQ-1 generated trigger; giving a long-term direct cross-verification of both clock systems. First results indicate very good precision of $<0.3 \mathrm{~ns}$ relative jitter (analysis in progress).

\subsection{Calibration with a pointlike LED source}

Dedicated LED-calibration runs were performed with a powerful LED located $\sim 100$ m outside the HiSCORE-9 array, generating simultaneous light flashes in all stations (see fig.7) - to calibrate residual time offsets (PMTs, FE-electronics), and to verify the relative time calibration and precision of the DAQ-1/2 systems. For both, good agreement was found, as shown for WR in fig.8 for the fitted time-residuals; it gives an upper WR-precision limit of $0.45 \mathrm{~ns}$ [8].

\subsection{Operation with Air Shower}

A final verification of precise and stable timing operation is the full reconstruction of cosmicray air showers, registered in routine operation with HiS-9 for 2013/14 and HiS-28 (2014/15; in progress). Using the calibration from LED runs, reconstruction of the shower front direction and position is done for HiS-9 [2, 8]. Figure 9 displays a reconstructed shower event (based on WRtiming only); the fitted time-residual distribution indicates an upper limit for $\sigma_{W R}$ of $<0.5 \mathrm{~ns}$ [8].

\section{Summary}

We applied the new White Rabbit technology for sub-nsec precision time-synchronization of the HiSCORE DAQ-system in various construction phase setups. The harwdare solution is 
HiSCORE-9: LED calibration

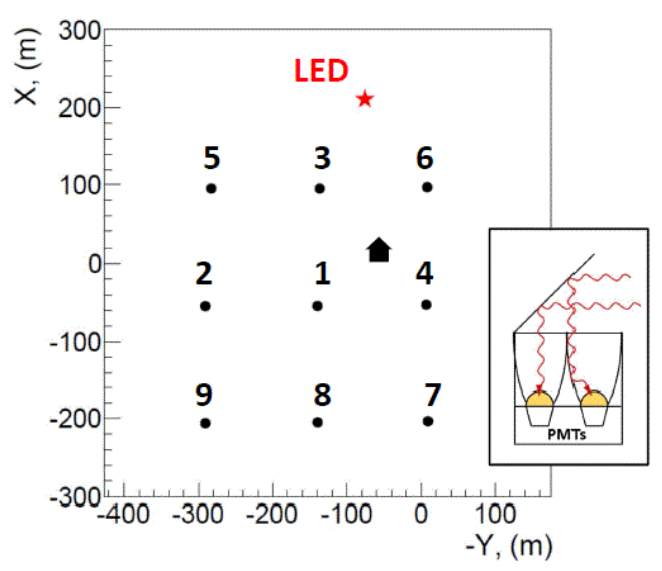

Figure 7: The HiS-9 array layout: Nine stations and LED source position (red star) for calibration runs; insert: station setup for calibration runs with $45^{\circ}$ inclined reflectors.
Fit Residuals: All stations

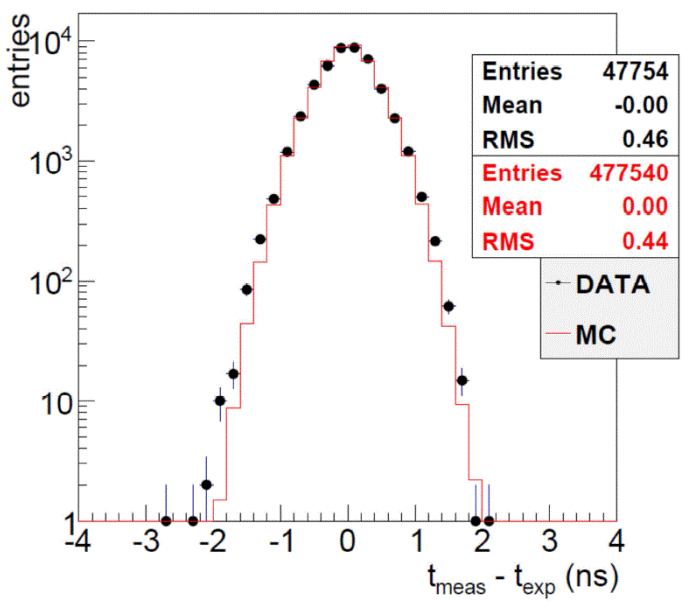

Figure 8: LED calibration: Distribution of fit residuals for LED events (all nine stations superimposed). Black dots: data; Red line: simulated events.

based on standard WR-components (SPEC, DIO5Ch and WRS), with HiSCORE-specific firmware developed for nsec-time-stamping, triggering, DAQ-control; and with support for WR monitoring.

Laboratory and special field setups yielded a timing-precision $\sigma_{W R}$ of better than $0.2 \mathrm{~ns}(<60 \mathrm{ps}$ in table-top setups). We gained long-term field experience with the HiSCORE-9 array, with WR embedded into the full DAQ, and routinely recoreded cosmic-ray data from 2013-2015. From external LED-calibration and air-shower reconstruction we obtain an upper limit for the WR-precision $\sigma_{W R}$ of better than $0.5 \mathrm{~ns}$ (likely driven by dominant non-WR systematics: hardware, air-shower). An ongoing, direct harwdare test installed with HiSCORE-28 indicates $\sigma_{W R}<0.3 \mathrm{~ns}$ under real life conditions.

We emphasize an advantage of the WR-architecture, beyond precision timing: digital, fully calibrated times are instantaneously available at the front-ends, which (1) significantly simplifies the DAQ, and (2) allows for digital trigger concepts based on nsec timing and next-neighbor/array topologies - which can be fast, complex, and yet simple to design [9]. Last not least: interfacing a given experiment to WR can be kept as simple as designing a (passive) mezzanine-interface card. Precision, stability and overall system performance of the White-Rabbit based timing makes it a prime candidate for next generation large scale experiments like CTA [4].

Acknowledgment: We acknowledge very helpful discussions, technical advices and support from the White Rabbit community during all phases of this work. The technical support from both SevenSolutions and Creotech was very helpful, too. We acknowledge the financial support from the Helmholtz association (grant HRJRG-303), that allowed to start this work.

\section{References}

[1] Tluczykont, M. et. al., DOI: 10.1016/j.astropartphys.2014.03.004 


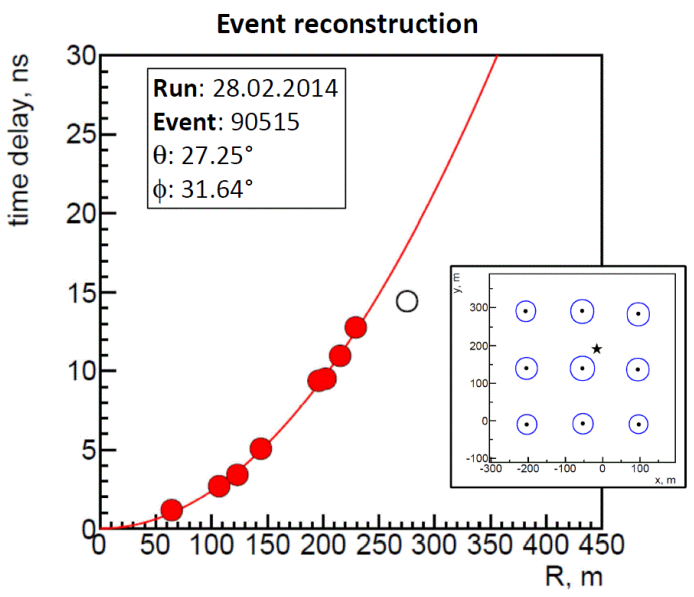

(a)

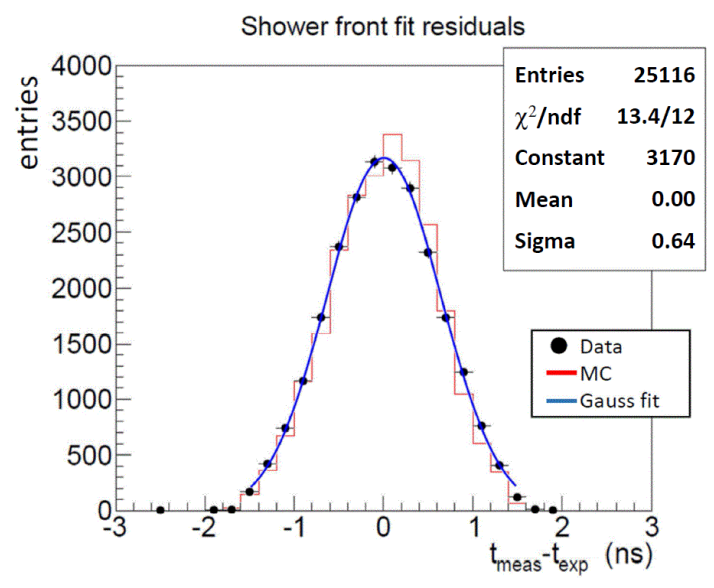

(b)

Figure 9: EAS shower reconstruction. (a) Arrival time delay vs distance R from the shower axis; for an event. Red/white dots: stations retained / excluded in the final fit; red line: reconstructed shower profile. Small panel: Reconstructed core position (black star), the area of the circles is proportional to $\log A$, with $A$ the station signal amplitude. (b) Distribution of fit residuals after shower reconstruction. Black dots: data; Red line: simulated events; Blue line: gaussian data fit.

[2] Budnev, N. et al, The Tunka detector complex: from cosmic-ray to gamma-ray astrophysics, Proceed. ECRS-2014, Kiel, 2014

[3] J.Serrano et al., The White Rabbit Project, ICALEPCS 2009, http://accelconf.web.cern.ch/accelconf/icalepcs2009/papers/tuc004.pdf

[4] Oya, I. et al, Status and plans for the array control and data acquisition system of the Cherenkov Telescope Array, contribution to ICRC2105

[5] M.Brückner and R.Wischnewski, A White Rabbit setup for sub-nsec synchronization, timestamping and time calibration in large scale astroparticle physics experiments, Proceed. ICRC-2013, Rio de Janeiro, ID-1146

[6] WR-supplier: SevenSols, Spain, http://www.sevensols.com; Creotech, Poland, http://www.creotech.pl

[7] M.Brückner et al., Results from the WhiteRabbit sub-nsec time synchronization setup at HiSCORE-Tunka, Proceed. ICRC-2013, Rio de Janeiro, ID-1158

[8] Porelli, A. et al, Timing calibration and directional reconstruction for Tunka-HiSCORE, Proceed. ECRS-2014, Kiel, 2014

[9] Shayduk, M. et al, A concept of wide-angle Cherenkov gamma-ray instrument with minimal imaging, contribution to ICRC2105, ID-0536; Shayduk, M. et al, A long buffer readout system for large-area Gamma-ray facilities, contribution to ICRC2105, paper-ID550

[10] Tunka-HiSCORE-Collaboration, Status of the HiSCORE experiment, Proceed ICRC-2013, Rio de Janeiro, ID-1164

[11] M.Wahl, H.J.Rahn, T.Röhlicke et al., Scalable time-correlated photon counting system with multiple independent input channels, Review of Scientific Instruments, Vol.79, 123113 (2008) 